Research Paper

\title{
LefI Contributes to the Differentiation of Bulge Stem Cells by Nuclear Translocation and Cross-Talk with the Notch Signaling Pathway
}

\author{
Yi Zhang ${ }^{* \bowtie}$, Jin $\mathrm{Yu}^{*}$, Chunying Shi, Yaqin Huang, Yun Wang, Tian Yang, Jin Yang \\ Department of Cell Biology, Third Military Medical University, Chongqing 400038, China \\ * These authors contributed equally.
}

$\square$ Corresponding author: Yi Zhang, Department of Cell Biology, Third Military Medical University, Chongqing, China. Tel: +8623 68752264; Fax: +86 23 68753260. E-mail: zhangyi@tmmu.edu.cn (Y. Zhang).

(c) Ivyspring International Publisher. This is an open-access article distributed under the terms of the Creative Commons License (http://creativecommons.org/ licenses/by-nc-nd/3.0/). Reproduction is permitted for personal, noncommercial use, provided that the article is in whole, unmodified, and properly cited.

Received: 2012.12.1I; Accepted: 2013.04.10; Published: 2013.04.17

\begin{abstract}
Lymphoid enhancer binding factor-I (Lefl) is an essential regulatory protein in the Wnt signal pathway, which controls cell growth and differentiation. Investigators in the field of skin biology have confirmed that multipotent bulge stem cells (BSCs) are responsible for hair follicle development and regeneration. However, the role of LefI remains poorly understood. In this study, we investigated the pattern of Lefl expression at different stages of the hair growth cycle. Lefl was strongly expressed during anagen but attenuated in both catagen- and telogen-phase hair follicles in vivo. When stem cells were induced to differentiate toward a hair fate in a co-culture system, LefI was notably up-regulated and accumulated in the nucleus, appearing to activate the target protein c-myc and jagged I. Simultaneously, the Wnt and Notch signaling pathways were co-activated, as confirmed by the increased expression of $\beta$-catenin and notchl. Plasmids expressing Lefl and $\Delta \mathrm{NLefl}$, a construct in which the $\beta$-catenin-binding domain of Lefl was deleted, were used to evaluate the effects of LefI on stem cell differentiation. Lefl overexpression promoted bulge stem cell differentiation toward a hair fate, which was accompanied by the subsequent migration of $\beta$-catenin into the nucleus, whereas no changes were observed in the control group. Taken together, our results demonstrate that Lefl plays an important role in bulge stem cell differentiation, promoting $\beta$-catenin translocation into the nucleus, activating downstream signaling molecules, eventually causing hair follicle bulge stem cells to adopt the hair fate.
\end{abstract}

Key words: Lef1; Hair follicle; Bulge stem cells; Wnt signaling; Differentiation

\section{Introduction}

It has been shown that the bulge region of the upper hair follicle is a repository of multipotent stem cells. These cells, called bulge stem cells (BSCs) or hair follicle stem cells (HFSCs), are normally slow-cycling but can be stimulated to proliferate in response to injury or to certain growth stimuli. Moreover, bulge stem cells can give rise to different epithelial cell types of hair follicles and participate in the formation of hair follicles, epidermis and sebaceous glands. Hair folli- cles are cyclical structures that undergo growth (anagen), regression (catagen) and rest (telogen) phases [1-2]. In 1990, Cotsarelis et al. proposed the "Bulge Activation Hypothesis", which states that the signals emitted from the dermal papillae trigger hair follicle growth [3]. Accumulated evidence has confirmed that the initiation of a new hair cycle relies on the activation of quiescent stem cells to proliferate and differentiate in response to signals from the dermal 
papilla (DP). Recent studies have shown that Wnt signals, including wnt $10 \mathrm{~b}$ and $\beta$-catenin, sustain DPs with their hair follicle induction activity [4-5]. These results support the importance of $\mathrm{Wnt} / \beta$-catenin signaling derived from the dermal papillae in maintaining the normal hair cycle.

In fact, Wnt signaling plays a crucial role in development and tumorigenesis and also controls self-renewal, proliferation and differentiation in many types of stem cells. The activation of this pathway appears to depend on the translocation of $\beta$-catenin from the cytoplasm to the nucleus and on interactions with transcription factors of the Lef1/Tcf family, which eventually regulate the transcription of target genes. Increasing evidence indicated that the Wnt signaling pathway was responsible for the morphogenesis and cycle maintenance of the hair follicle. Hair follicle development was hindered by the conditional ablation of $\beta$-catenin or overexpression of the Wnt inhibitor Dkk during embryogenesis [6-7]. In contrast, the overexpression of these components might produce an excess of hair follicles or even produce hair follicle tumors [7-8]. As most of the previous studies of Wnt signaling and hair follicle biology have focused on $\beta$-catenin, the possible role of lymphoid enhancer factor-1 (Lef1) in follicle development is not well known. In neural progenitor cells, the inhibition of Lef1 decreased cell proliferation, resulting in a reduction of midbrain tectum size [9]. In early 1994, Genderen et al. found that limiting Lef1 by gene knockdown resulted in sparse hair, with a complete loss of whisker follicles and the secondary follicles that account for the bulk of the hair coat [10]. Subsequently, investigators revealed that Lef1 was present during skin embryogenesis in the ectoderm and led to hair development [11]. These results imply that Lef1 is functionally required for hair morphogenesis.

Experimental evidence has supported the role of Wnt signaling components in the differentiation processes of epidermal stem cells. For example, $\beta$-catenin-deficient stem cells fail to differentiate into follicular keratinocytes and instead adopt an epidermal fate [12]. Nguyen et al. found that Tcf3 might function to direct embryonic skin progenitors along their hair fate. Their subsequent work discovered that coexpress of Tcf3 and Tcf 4 was response for long-term maintenance and wound repair of both epidermis and hair follicles $[13,14]$. Recently, the presence and localization of Lef- 1 in murine hair follicles during the first hair cycle were investigated [15], but the mechanism of this process is still under study. To better understand the possible functions of Lef1 in hair follicle biology, we characterized the expression pattern of Lef1 during the postnatal hair follicle cycle and eval- uated the changes of Lef1 in differentiated bulge stem cells. Our data indicated that Lef1 was critical to the differentiation of bulge stem cells and that the overexpression of Lef1 in bulge stem cells might promote the translocation of $\beta$-catenin into the nucleus to activate downstream molecules in the Wnt signaling pathway. Furthermore, we also investigated the role of intercellular signaling mediated by Notch in the process of how bulge stem cells were prompted to select a hair fate.

\section{Materials and Methods}

\section{Tissue preparation}

Hair follicles representing early anagen, anagen, catagen, telogen and late telogen were collected from 7-, 15-, 22-, 28- and 32-day-old SD rats (Animal Center, Third Military Medical School, Chongqing, China), respectively. After fixation and dehydration, the rat skin tissues were embedded in paraffin, and serial sections $(5 \mu \mathrm{m})$ were cut using a cryostat (Leica).

\section{Cell culture}

The hair follicles were isolated by microdissection, and the bulb and the bulge region were microdissected, as previously described [16]. To isolate the epithelial stem cells, the bulge region was incubated in $0.25 \%$ dispase (Sigma) for $30 \mathrm{~min}$ at $4^{\circ} \mathrm{C}$ and then treated with $0.25 \%$ trypsin (Hyclone) for $15 \mathrm{~min}$ at $37^{\circ} \mathrm{C}$. The cell suspension was collected and maintained in DMEM/F12 medium at $37^{\circ} \mathrm{C}$ in a $5 \% \mathrm{CO}_{2}$ atmosphere. The bulbs were placed in $0.2 \%$ collagenase (Sigma) for 3-5 h until the separation of DPs from the matrix was observed. After the supernatant was removed by centrifugation, the harvested dermal papillae were plated in DMEM/F12 medium containing 10\% fetal bovine serum (Invitrogen).

\section{Establishment of the induction microenvi- ronment in vitro}

Two systems were designed to induce the bulge stem cells to differentiate directly into a hair fate. The bulge stem cells were incubated in DP-conditioned medium (DP-CM) or co-cultured with DP cells using Transwell devices. In brief, the bulge stem cells were first plated in 6-well plates, and the Transwell inserts were then added and seeded with DP cells in the inner compartment. All the cells were incubated with fresh DMEM/F12 medium, and the bulge stem cells were later harvested for further experiments.

\section{Immunohistochemical and immunofluores- cent staining}

Tissue sections were deparaffinized with xylene and rehydrated in graded ethanol. The cells were 
plated on glass coverslips and fixed in ice-cold acetone. The speciments were then blocked with $1 \%$ $\mathrm{H} 2 \mathrm{O} 2$ and $10 \%$ goat serum (Sigma) and subsequently incubated with anti-Lef1 (1:100, Santa Cruz), anti-Keratin6 (1:100, Santa Cruz) or anti-S100A3 (1:100, Santa Cruz) antibodies overnight at $4^{\circ} \mathrm{C}$. The immunohistochemical assays were performed according to the manufacturer's recommendations, and staining was visualized by treatment with diaminobenzidine (DAB; Beijing Zhongshan Biotechnology Co.). The slides were then viewed using an optical microscope (Leica). Percentages of differentiated cells were calculated from images of randomly selected fields for each cell population.

In other experiments, the bulge stem cells were analyzed by immunofluorescent staining. After fixing and blocking, as described above, the cells were incubated with the following diluted primary antibodies: anti-Lef1 (1:100, Santa Cruz), anti- $\beta$-catenin (1:100, Santa Cruz), anti-a6-integrin (1:100, Abcam) or anti-CD34 (1:100, Santa Cruz). The cells were then incubated with the appropriate fluorescein isothiocyanate-conjugated secondary antibodies and counterstained with 4',6-diamidino-2-phenylindole (DAPI) (Sigma). The sections were visualized using a fluorescence microscope (Nikon).

\section{Real-Time PCR}

Total RNA was isolated from cultured cells using the RNeasy kit (Qiagen). cDNAs were synthesized from $2 \mu \mathrm{g}$ of cytosolic RNA with a cDNA kit (Bio-Rad) according to the manufacturer's protocol. The realtime PCR was carried out with a CFX96 Detection System (Bio Rad). In each experiment, the GAPDH housekeeping gene was amplified as a reference standard. The primer sequences were described as follow: 5' AGGGCGACTTAGCAGACATCAA 3' and 5' TGTAGCCAGAGTAACTGGAGTAGGAG $3^{\prime}$ 'for Lef- $1, \quad 5^{\prime}$ GCCAAAAGGGTCATCATC $3^{\prime}$ and $5^{\prime}$ ATGACCTTGCCCACAGCCTT $3^{\prime}$ for GAPDH. All PCR reactions were performed in duplicate of three independent experiments.

\section{Plasmid construction and transient transfec- tions}

According to the procedure described previously [17], the Lef1 and $\Delta$ NLef1 genes were amplified from the cDNA of newborn rat kidneys. After purification, the target genes were digested using EcoRI and KpnI (Roche) and then ligated into pEGFP-C1. The success of the ligation was confirmed by EcoRI and KpnI double digestion and DNA sequencing. The hair follicle bulge stem cells were grown in OPTI-MEM medium (Gibco), and the transfections were performed with Lipofectamine 2000 (Invitrogen) according to the manufacturer's protocol. After $48 \mathrm{~h}$ of transfection, the cells were harvested for further immunofluorescence assays or western blotting.

\section{Western blot analysis}

Equal amounts of protein lysates were subjected to SDS-PAGE and transferred to a PVDF membrane by electroblotting. The antibodies against Lef1 (1:2000 dilution), $\beta$-catenin (1:2000 dilution), c-myc (1:1000 dilution), jagged1 (1:3000 dilution) and notch1 (1:1000 dilution) were used. A $\beta$-actin antibody at a 1:10000 dilution was used as a loading control. The protein bands were visualized using an imaging system (Bio-Rad).

\section{Luciferase assay}

TOPFlash and FOPFlash are widely used to evaluate the activation of $\beta$-catenin/Lef1 signaling. After transient transfection with the TOPFlash and FOPFlash plasmids, the cells were incubated for $24 \mathrm{~h}$, and the luciferase activity in the cell lysate was determined using a luminometer. The Luciferase Reporter Assay System was purchased from Promega.

\section{Statistical analysis}

All the results were expressed as mean \pm standard deviation (SD) at least three independent experiments. Microsoft Excel was used for statistical analyses. The statistical significance of the differences was evaluated by paired t-test, and $\mathrm{p}<0.05$ was considered statistically significant.

\section{Results}

\section{Lefl expression is dynamic during the hair fol- licle cycle}

Although Lef1 is known to be induced in the pluripotent ectoderm and underlying mesenchyme during the embryonic development of the hair follicle [18-19], its precise expression pattern during the postnatal hair follicle cycle has not yet been characterized. In this study, immunohistochemical examination demonstrated that the expression of the Lef1 protein was dynamic throughout the follicle cycle (Fig. 1A). During anagen, the stage at which the bulge stem cells are activated, migrate downward and initiate hair formation, strong nuclear Lef1 expression was detected in the bulge, matrix and outer root sheath (ORS), which contain the proliferating and differentiating epithelial cells. Subsequently, the level of Lef1 expression decreased in catagen and telogen, which represent the regressive and quiescent phases of the hair cycle, respectively. During these stages, positive staining was only weakly detected in the inner root 
sheath (IRS) and not in the ORS or bulb. However, at the early anagen of the next hair cycle, Lef1 was strongly expressed in the entire hair follicle. These results indicated that Lef1 might play an important role in the cyclic growth of the hair follicle. In addition, during late telogen, characterized by the transition from telogen to anagen as quiescent stem cells are were activated, the Lef1 protein again accumulated in the bulge and matrix areas (Fig. 1B). These results suggested that Lef1 participates in the process of bulge stem cell re-activation and the initiation of a new round of hair growth.
A

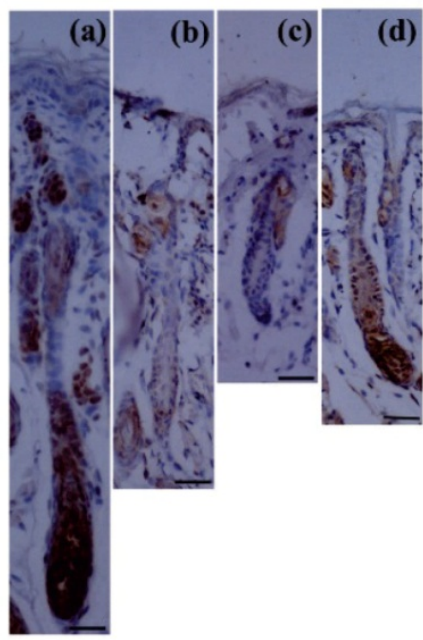

B

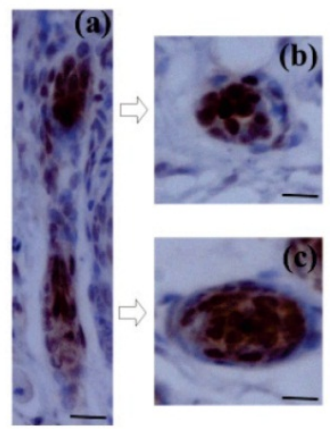

Figure I. Expression of LefI in the cycle of hair follicle. (A) Immunohistochemical staining of Lefl at anagen (a), catagen (b),

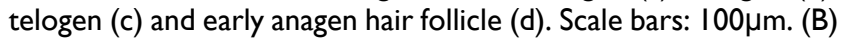
The location of LefI protein in late telogen hair follicles. Scale bars: (a) $50 \mu \mathrm{m},(\mathrm{b}-\mathrm{c}) 20 \mu \mathrm{m}$.

\section{Bulge stem cells are prompted to adopt a hair fate via DP induction}

To establish an induction model, bulge stem cells and DP cells were isolated and cultured in vitro. The primary bulge stem cells grew clonally and exhibited a round, slabstone-shaped appearance. Immunofluorescent staining showed that the specific stem cell markers CD34 and a6-integrin were strongly expressed in the bulge stem cells (Fig. 2A). A Transwell system and DP-conditioned medium were used to evaluate the possibility that the bulge stem cells underwent differentiation, as described in the Materials and Methods. As anticipated, the morphology of the resulting cells changed after $48 \mathrm{~h}$ of incubation, with the appearance of a larger volume and higher nuclear-cytoplasmic ratio (Fig. 2B). Furthermore, the expression levels of hair follicle-specific markers were examined in all the groups. Keratin6 and S100A3 were both notably up-regulated in the DP-treated cells compared with the untreated cells, and the exposure of the bulge stem cells to DP led to a pronounced increase in the keratin6-positive and S100A3-positive cell populations. Furthermore, no Oil Red O-positive cells were observed after DP treatment (data not shown). All these results support the hypothesis that bulge stem cells commit to a hair fate in response to DP induction.

$\mathbf{A}$

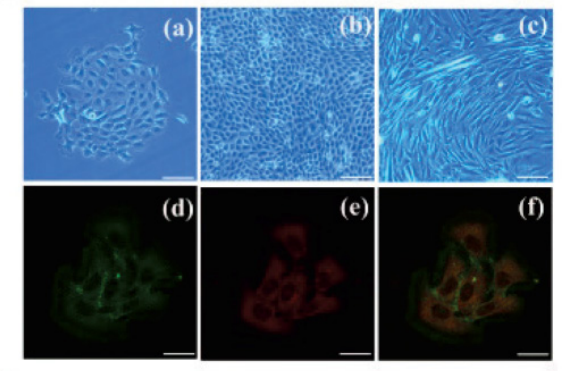

B

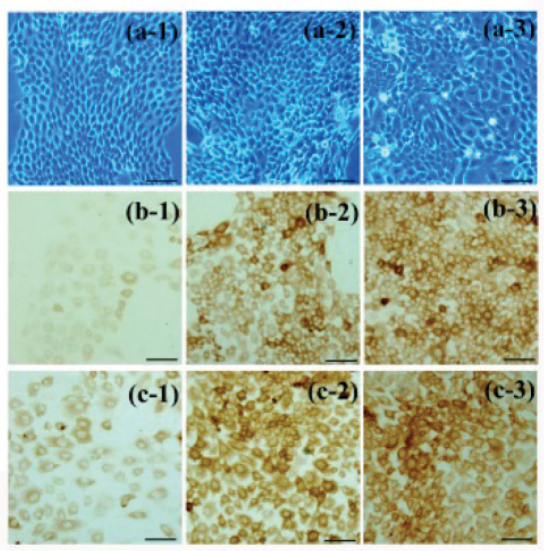

Figure 2. Differentiation of bulge stem cells on the condition of DP cells induction. (A) The bulge stem cells (a: primary; b: passage) and DP cells(c) are isolated and cultured in vitro. Immunofluoresence staining shows a6-integrin (d) and CD34 (e) expression. Merge indicates an overlap of both antibodies ( $f$ ). Scale bar: (a-c) $50 \mu \mathrm{m},(\mathrm{d}-\mathrm{f}) 20 \mu \mathrm{m}$. (B) Charecteristic of differentiated bulge stem cell is detected after DP induction, showing the morphologic features (a), keratin 6 (b) and SI00A3 (c) expression of bulge stem cells. (I) control group; (2) DP-CM group; (3) co-culture group. Scale bars: $50 \mu \mathrm{m}$.

\section{Lefl promotes the differentiation of bulge stem cells by activating the Wnt signaling pathway in vitro}

Lef1 is known as a key member in Wnt signaling, functioning in cell proliferation and differentiation by triggering the activity of downstream genes. To examine whether Lef1 was activated in bulge stem cells after stimulation with DP cells, the expression of Lef1 and its associated factors such as $\beta$-catenin, c-myc and 
jagged1 were measured. Our data showed that DP elevated the expression of Lef1 in bulge stem cells (Fig. 3A), suggesting that Lef1 was involved in the differentiation of bulge stem cells toward a hair fate in vitro. Previous evidence has demonstrated that the accumulation of nuclear Lef1 might be responsible for Wnt activation. This hypothesis was supported by the results that the induced cells exhibited strong nuclear staining of Lef1 (Fig. 3B, and C). To further confirm the activation of the Wnt pathway, a TOP/FOP luciferase activity analysis was performed. After transfected with the TOP/FOP luciferase reporter plasmid, the DP-induced cells showed 5- or 6-fold higher luciferase activities than the non-induced cells (Fig. 3D).

Similarly, western blotting showed a similar in- crease in the expression of $\beta$-catenin and c-myc when Lef1 was up-regulated in both co-culture and the medium-induced model. Interestingly, high jagged1 and notch1 expression were also detected in the induced cells (Fig. 4A). Thus, we investigated whether the differentiation of induced bulge cells was mediated through the Notch pathway. As shown in Fig. 4B, treatment with DAPT, $\gamma$-secretase inhibitor to block the Notch pathway, substantially led to the suppression of cell differentiation in the co-culture and DP-CM condition. These data not only suggested that Lef1 might regulate bulge stem cell differentiation in a Wnt-dependent manner, but also indicated the essential role of Notch signaling in the promotion of stem cell activation.
A

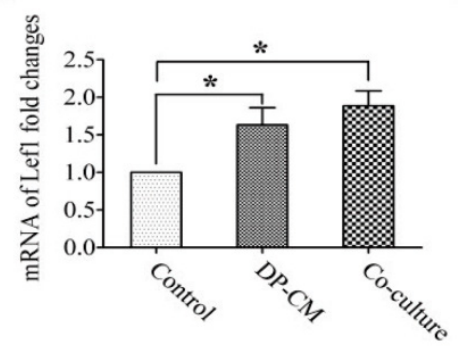

B

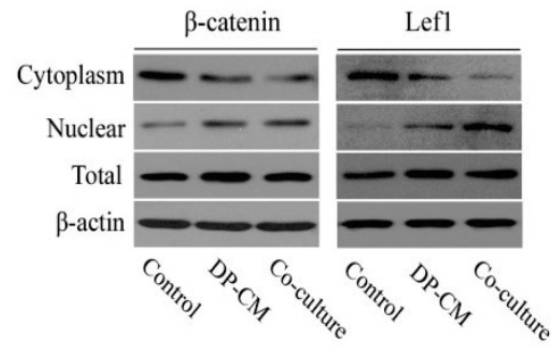

C

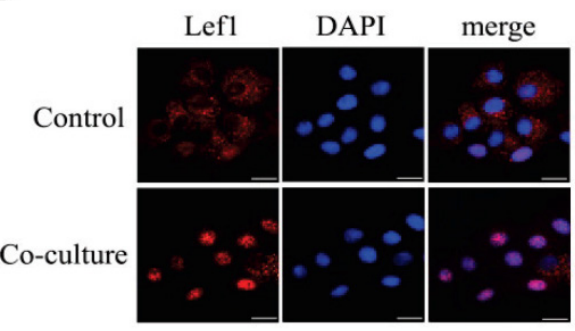

D

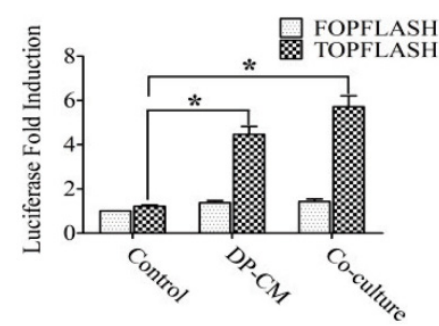

Figure 3. Lefl promoted cell differentiation via Wnt signaling pathway. (A) LefI mRNA expression level measured by realtime PCR $(* p<0.05)$. (B) The cytoplasmic and nuclear expression of Lefl and $\beta$-catenin in the bulge stem cells treated with DP or not. (C) Immunofluorescence analysis on the location of Lefl in those cells. Scale bars: $20 \mu \mathrm{m}$. (D) The transcriptional activity of Wnt signaling was determined by luciferase reporter assay. $\left({ }^{*} p<0.05\right)$. Data shown here are representative for three experiments.

A

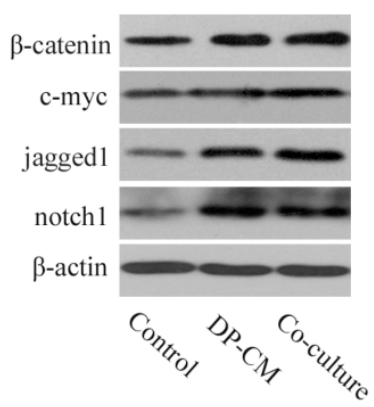

B

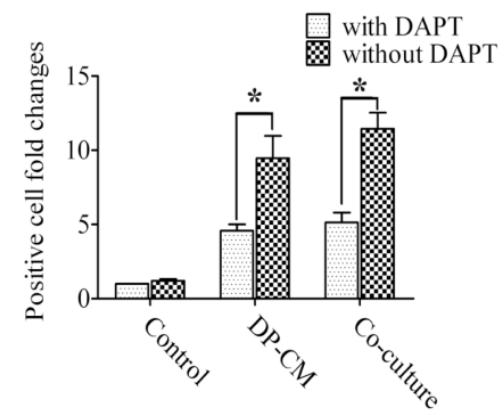

Figure 4. Notch signaling is involved in the differentiation of bulge stem cells by induction with DP. (A) A western blot analysis of $\beta$-catenin, c-myc, jaggedI and notchl expression was detected in differentiated cells. (B) The suppressive effect of DAPT on cell differentiation was comparatively analysed by the folds of positive cells in DP-mediated condition after treatment with DAPT or not $\left({ }^{*} p<0.05\right)$. Data shown here are representative for three experiments. 


\section{LefI mediates the nuclear import of $\beta$-catenin via direct binding}

To define the direct effect of Lef1 in the differentiation of bulge stem cells and to confirm whether $\beta$-catenin is transferred into the nucleus by binding to Lef1, we constructed plasmids to express wild-type Lef1 plasmid and a Lef1 construct lacking the $\beta$-catenin-binding domain ( $\Delta$ NLef1) [20-21]. Upon DP induction, the cells transfected with Lef1 exhibited enhanced keratin 6 and S100A3 expression, whereas only a weak change was observed in the $\Delta$ NLef1 group (Fig. 5A and B). These results provide evidence that Lef1 promoted the differentiation of bulge stem cells into a hair fate and interacted directly with $\beta$-catenin.

Because $\beta$-catenin translocation was required for the activation of the Wnt signaling pathway, the expression of $\beta$-catenin was also examined. No significant difference in total $\beta$-catenin was detected, but the nuclear accumulation of $\beta$-catenin was up-regulated more strongly in the cells that were transfected with Lef1 than in the $\Delta$ NLef1 transfection or control cells (Fig. 5C and D). These results suggested that Lef1 could effectively trigger $\beta$-catenin translocation from the cytoplasm and plasma membrane to the nucleus and that this coordination directly promoted bulge stem cell differentiation toward a hair fate.

To further investigate whether the Notch pathway participated in the Lef1-regulated cell differentiation, we tested the effect of suppressing Notch activ-

A

B

D
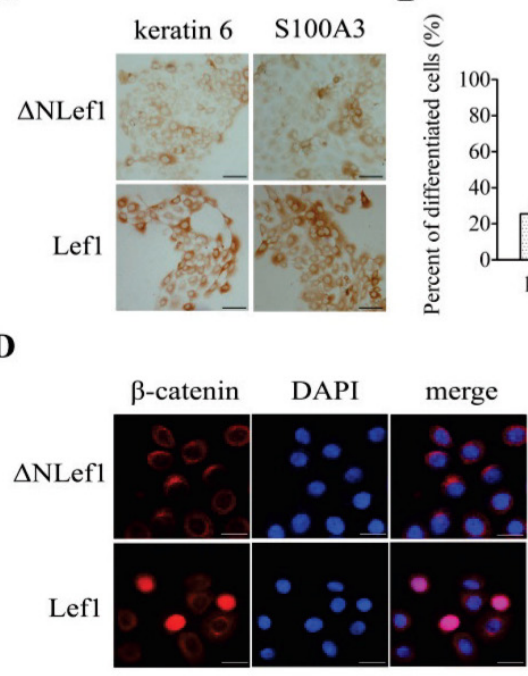

ity on cell differentiation by using DAPT. Results showed that treatment with DAPT significantly inhibited differentiation of the Lef1-transfected cells, whereas weakly to that of $\Delta$ NLef1-transfected cells (Fig. 5E). Taken together, these support that the role of Lef1 in stem cell differentiation is partly dependent on the Notch signaling pathway.

\section{Discussion}

Lef1, the key nuclear mediator of the Wnt signaling pathway, is highlighted for its roles in regulating the epithelial/mesenchymal interactions involved in mammary glands, teeth and hair follicles. Previous studies have revealed that the development of mammary glands and teeth was aborted in the absence of Lef1 [22-23]. Present studies on Lef1 in hair follicle biology have usually focused on hair development. Lef1 knockout led to a loss of vibrissal hair follicles and an arrest of dorsal hair follicles, whereas Lef1 activation induced de novo hair follicle formation [11]. Here, we provide the description of the dynamic expression of Lef1 during the hair cycle and demonstrate its role in the fate decision of bulge stem cells in vitro. We also report that Lef1 could bind to $\beta$-catenin directly and translocate it into the nucleus, resulting in the activation of the Wnt signaling pathway. Clarifying the molecular mechanisms of stem cell regulation and function will contribute greatly to understanding tissue homeostasis and the pathogenesis of various skin diseases [24].

\section{C}

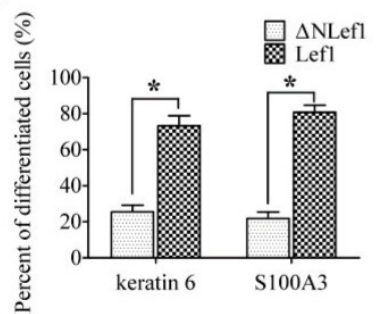

$\mathbf{E}$

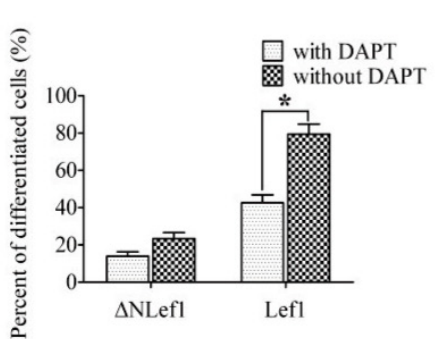

Figure 5. Analysis of bulge stem cells differentiation induced by LefI and $\Delta$ NLefl plasmid transfections. (A) $\Delta N L$ efland Lefl plasmid regulated cell differentiation in DP-mediated condition, detected with keratin 6 and SI00A3. Scale bars: 50 $\mu$ m. (B) The statistical analysis of the differentiating efficiency by evaluating the percentages of positive cells in DP-mediated condition. $\left({ }^{*} p<0.05\right)$. (C) Western blot analysis of the total $(T)$ and nuclear $(N) \beta$-catenin expression. (D) The nuclear transportation of $\beta$-catenin was observed after transfected with LefI or $\Delta$ NLefI plasmids. Scale bars: $20 \mu \mathrm{m}$. (E) The effect of Notch signaling on cell differentiation was analysed by SI00A3 expression $(* p<0.05)$. Data shown here are representative for three experiments. 
It has been generally believed that the stem cells in the bulge region are responsible for hair growth and regeneration, and also participate in the renewal of the epidermis and sebaceous glands. Thus, bulge stem cells are regarded as multipotent stem cells in the skin and an essential cellular resource of epidermis, hair follicles and sebaceous glands [25]. However, the mechanisms involved in bulge cell fate decision are not known. Evidence from studies of $\beta$-catenin has shown that the Wnt signaling pathway is indispensable for hair follicle morphogenesis. For example, the components of this pathway are expressed at a higher level during hair follicle development and participate in stem cell differentiation [5, 12]. Moreover, to examine the consequences of repressing beta-catenin/Lef1 signaling in mouse epidermis, Niemann et al. expressed a DeltaNLef1 transgene, which lacks the beta-catenin binding site, leading to differentiation of hair follicles into epidermal keratinocytes and skin tumor formation [17]. These suggest that beta-catenin/Lef1 signaling can determine the differential fate of bulge stem cells. Considering that Wnt signaling played an essential role in the biological processes of stem cells and that Lef1 was up-regulated during hair development, we investigated the expression of Lef1 during different phases of the hair follicle cycle. As shown in Fig. 1, Lef1 was active during anagen and attenuated in catagen and telogen, consistent with a function for Lef1 in hair growth. Simultaneously, we also demonstrated the elevated expression of nuclear Lef1 during anagen and late telogen. It is well known that quiescent stem cells are activated by adjacent DPs to produce new hair when the transition from telogen to anagen occurs. Our results suggest that Lef1 is a critical factor for the maintenance of the normal hair cycle and also might participate in the re-activation of bulge stem cells and regulate their decision of a hair fate.

Because multipotent bulge stem cells could be stimulated to differentiate by mesenchymal DP cells, which supply the specific hair-inducing signals required for hair outgrowth, we used a co-culture system in our study. Roh et al. had reported that the nuclear $\beta$-catenin levels were increased in DP-induced human epithelial stem cells. [26]. Similarly, we showed that DP cells also markedly promoted Lef1 up-regulation and translocation into the nucleus in differentiated cells. In the canonical Wnt signaling pathway, the activation of target genes usually depends on the binding of $\beta$-catenin to the TCF/LEF complex [27]. Using the TOP/FOP flash assay, we further showed that the transcriptional activation of the $\beta$-catenin/Lef1 complex was initiated after DP treatment. These results indicate that Lef1 plays a dominant role in the differentiation of bulge stem cells via the Wnt signaling pathway.

Our results indicated that the functions of Lef1 are dependent on the activation of specific target genes. Thus, we analyzed the expression of c-myc and jagged1 in induced bulge stem cells. C-myc is a target gene of $\beta$-catenin/Lef1 that participates in the regulation of cell cycle progression. Bull et al. confirmed that c-myc immunoreactivity in the bulge region of the outer root sheath correlated with the putative hair follicle stem cell compartment [28]. Supporting evidence was provided in a recent study from our lab in which c-myc inhibition significantly restrained the development of anagen hair follicles, whereas c-Myc overexpression promoted hair growth [29]. Consistent with these findings, we showed that the up-regulation of c-myc in bulge stem cells was tightly correlated with DP induction. This suggests that Lef1-mediated signaling might be responsible for determining cell fate by promoting the expression of c-myc.

Jagged1, the ligand of Notch signaling, is identified as another conserved target of the Wnt/ $\beta$-catenin signaling pathway. Its promoter region contains a Tcf/Lef binding site, and it functions as a key molecule in maintaining stem cell homeostasis [30]. Estrach et al. found that the deletion of jagged1 inhibited hair growth and prevented new hair follicle formation, revealing the interaction of the Wnt and Notch signaling pathways in regulating hair follicle cycle maintenance [31]. In general, Notch signaling plays a key role in regulating the self-renewal, proliferation, and differentiation of stem cells [32-34]. Previous studies have documented that Notch was widely expressed in developing or differentiating hairs, led to the aberrant differentiation of the hair cortex [35-36], and inhibited the terminal differentiation of the epidermis [37]. Here, we demonstrate an increase in the expression of jagged1 following the up-regulation of $\beta$-catenin and Lef1 induced by DP treatment. This observation indicated that jagged1 might act as a downstream target of the Wnt/ $\beta$-catenin pathway to regulate cell fate determination.

In fact, the activity of Wnt and Notch signaling is central to stem cell fate decisions during development. In previous studies focused on the interactions of these two signaling pathways, it has been proposed that Wnt and Notch signaling should create an integrated molecular mechanism to switch a cell state [38]. Based on this, we further showed the increased notch1 activity in differentiating bulge stem cells. These findings indicate that $\mathrm{Wnt} / \beta$-catenin signaling is involved in the cell fate determination of bulge stem cells by crosstalk with the activation of the Notch pathway. However, Shahi et al. demonstrated that the 
Wnt and Notch pathways had interrelated but opposing roles in prostate progenitor cell proliferation and differentiation [39]. Therefore, further studies are necessary to better characterize this complex signaling network.

In the canonical Wnt signaling pathway, the activation of target genes usually depends on the translocation of $\beta$-catenin and Lef1 into the nucleus. It is generally accepted that Lef1 could be transported into the nucleus with the aid of importin or in a Ran-independent manner [40]; however, the nuclear import of $\beta$-catenin might be independent of the nuclear pore complex receptor importin- $\alpha / \beta$ [41]. There is evidence that $\beta$-catenin can act as a nuclear import receptor to induce the nuclear translocation of Lef1 [42], indicating a close interaction between Lef1 and $\beta$-catenin. Furthermore, the overexpression of Lef1 in epidermal cells and tumor cells was able to promote the nuclear translocation of $\beta$-catenin in vitro [43-44]. Consistent with this observation, we found significant nuclear accumulation of $\beta$-catenin in the Lef1 plasmid-transfected cells but not in cells transfected with a Lef1 mutant lacking the $\beta$-catenin binding domain. These findings indicate that the additional activation of Lef1 is helpful to promote the nuclear translocation of $\beta$-catenin by direct binding. This hypothesis is further confirmed by the presence of $\beta$-catenin and Lef1 in the nucleus during the process of bulge stem cell differentiation.

In summary, we found that Lef1 droved bulge stem cell differentiation directly toward a hair fate and promoted the nuclear translocation of $\beta$-catenin. In addition, Notch signaling also appeared to be activated upon cell fate determination by collaborating with Wnt pathway. These results suggested that Lef1 played important roles in stem cell processes; further characterization and functions of this protein would be required for a complete understanding of the mechanism of stem cell differentiation.

\section{Acknowledgments}

This work was supported by the grants of National Nature Science Foundation of China (No. 30900737).

\section{Competing Interests}

The authors have declared that no competing interest exists.

\section{References}

1. Alonso L, Fuchs E. The hair cycle. J Cell Sci. 2006; 119(Pt 3):391-3.

2 Ohyama M, Zheng Y, Paus R, et al. The mesenchymal component of hair follicle neogenesis: background, methods and molecular characterization. Exp Dermatol. 2010; 19:89-99.
3. Cotsarelis G, Sun TT, Lavker RM. Label-retaining cells reside in the bulge area of pilosebaceous unit: implications for follicular stem cells, hair cycle, and skin carcinogenesis. Cell. 1990; 61:1329-37.

4. Ouji Y, Ishizaka S, Yoshikawa M. Dermal papilla cells serially cultured with Wnt-10b sustain their hair follicle induction activity after transplantation into nude mice. Cell Transplant. 2012; 21:2313-24.

5. Soma T, Fujiwara S, Shirakata $Y$, et al. Hair-inducing ability of human dermal papilla cells cultured under $\mathrm{Wnt} / \beta$-catenin signalling activation. Exp Dermatol. 2012; 21:307-9.

6. Sick S, Reinker S, Timmer J, et al. WNT and DKK determine hair follicle spacing through a reaction-diffusion mechanism. Science. 2006; 314:1447-50.

7. Ito M, Yang Z, Andl T, et al. Wnt-dependent de novo hair follicle regeneration in adult mouse skin after wounding. Nature. 2007; 447:316-20.

8. Lo Celso C, Prowse DM, Watt FM. Transient activation of beta-catenin signalling in adult mouse epidermis is sufficient to induce new hair follicles but continuous activation is required to maintain hair follicle tumors. Development 2004; 131:1787-99.

9. Ota $S$, Ishitani $S$, Shimizu $N$, et al. NLK positively regulates Wnt/ $\beta$-catenin signalling by phosphorylating LEF1 in neural progenitor cells. EMBO J. 2012; 31:1904-15.

10. van Genderen C, Okamura RM, Fariñas I, et al. Development of several organs that require inductive epithelial-mesenchymal interactions is impaired in LEF-1-deficient mice. Genes Dev. 1994; 8:2691-703.

11. Merrill BJ, Gat U, DasGupta R, et al. Tcf3 and Lef1 regulate lineage differentiation of multipotent stem cells in skin. Genes Dev. 2001; 15:1688-705.

12. Huelsken J, Vogel R, Erdmann B, et al. $\beta$-catenin controls hair follicle morphogenesis and stem cell differentiation in the skin. Cell. 2001;105:533-45.

13. Nguyen H, Rendl M, Fuchs E. Tcf3 governs stem cell features and represses cell fate determination in skin. Cell. 2006;127:171-83.

14. Nguyen H, Merrill BJ, Polak L, et al. Tcf3 and Tcf4 are essential for long-term homeostasis of skin epithelia. Nat Genet. 2009; 41:1068-75.

15. Wang HD, Yang L, Yu XJ, et al. Immunolocalization of $\beta$-catenin and Lef-1 during postnatal hair follicle development in mice. Acta Histochem. 2012; 114:773-8.

16. Zhang $\mathrm{Y}$, Xiang $\mathrm{M}$, Wang $\mathrm{Y}$, et al. Bulge cells of human hair follicles: segregation, cultivation and properties. Colloids Surf B Biointerfaces. 2006; 47:50-56.

17. Niemann C, Owens DM, Hülsken J, et al. Expression of DeltaNLef1 in mouse epidermis results in differentiation of hair follicles into squamous epidermal cysts and formation of skin tumours. Development. 2002;129:95-109.

18. DasGupta R, Fuchs E. Multiple roles for activated LEF/TCF transcription complexes during hair follicle development and differentiation. Development. 1999; 126:4557-68.

19. Liu X, Driskell RR, Luo M, et al. Characterization of Lef1 promoter segments that facilitate inductive developmental expression in skin. J Invest Dermatol. 2004; 123:264-74.

20. Arce L, Yokoyama NN, Waterman ML. Diversity of LEF/TCF action in development and disease. Oncogene. 2006; 25:7492-504.

21. Wang W, Ji P, Steffen B, et al. Alterations of lymphoid enhancer factor-1 isoform expression in solid tumors and acute leukemias. Acta Biochim Biophys Sin (Shanghai). 2005; 37:173-80.

22. Kratochwil K, Galceran J, Tontsch S, et al. FGF4, a direct target of LEF1 and Wnt signaling, can rescue the arrest of tooth organogenesis in Lef1(-/-) mice. Genes Dev. 2002; 16: 3173-85.

23. Boras-Granic $K$, Chang $H$, Grosschedl $R$, et al. Lef1 is required for the transition of Wnt signaling from mesenchymal to epithelial cells in the mouse embryonic mammary gland. Dev Biol. 2006; 295: 219-31.

24. Petersson M, Niemann C. Stem cell dynamics and heterogeneity: implications for epidermal regeneration and skin cancer. Curr Med Chem. 2012; 19:5984-92.

25. Oshima H, Rochat A, Kedzia C, et al. Morphogenesis and renewal of hair follicle from adult multipotene stem cells. Cell. 2001; 104:233-45.

26. Roh C, Tao Q, Lyle S. Dermal papilla-induced hair differentiation of adult epithelial stem cells from human skin. Physiol Genomics. 2004; 19:207-17.

27. MacDonald BT, Tamai $\mathrm{K}, \mathrm{He} \mathrm{X}$. Wnt/beta-catenin signaling: components, mechanisms, and diseases. Dev Cell. 2009; 17:9-26.

28. Bull JJ, Müller-Röver S, Patel SV, et al. Contrasting localization of c-Myc with other Myc superfamily transcription factors in the human hair follicle and during the hair growth cycle. J Invest Dermatol. 2001;116: 617-22. 
29. Wang N, Yang T, Li J, et al. The expression and role of c-Myc in mouse hair follicle morphogenesis and cycling. Acta Histochem. 2012; 114:199-206.

30. Katoh M, Katoh M. Notch ligand, JAG1, is evolutionarily conserved target of canonical WNT signaling pathway in progenitor cells. Int J Mol Med. 2006; 17:681-5.

31. Estrach S, Ambler CA, Lo Celso C, et al. Jagged 1 is a beta-catenin target gene required for ectopic hair follicle formation in adult epidermis. Development. 2006; 133:4427-38

32. Fox V, Gokhale PJ, Walsh JR, et al. Cell-cell signaling through NOTCH regulates human embryonic stem cell proliferation. Stem Cells.2008; 26:715-23.

33. Wen Y, Bi P, Liu W, et al. Constitutive Notch activation upregulates Pax7 and promotes the self-renewal of skeletal muscle satellite cells. Mol Cell Biol. 2012; 32:2300-11.

34. Torres J, Prieto J, Durupt FC, et al. Efficient differentiation of embryonic stem cells into mesodermal precursors by BMP, retinoic acid and Notch signaling. PLoS One. 2012; 7:e36405.

35. Favier B, Fliniaux I, Thélu J, et al. Localisation of members of the notch system and the differentiation of vibrissa hair follicles: receptors, ligands, and fringe modulators. Dev Dyn. 2000; 218:426-37.

36. Lin MH, Leimeister C, Gessler M, et al. Activation of the Notch pathway in the hair cortex leads to aberrant differentiation of the adjacent hair-shaft layers. Development. 2000; 127:2421-32.

37. Rangarajan A, Talora C, Okuyama R, et al. Notch signaling is a direct determinant of keratinocyte growth arrest and entry into differentiation. EMBO J. 2001; 20:3427-36.

38. Muñoz-Descalzo S, de Navascues J, Arias AM. Wnt-Notch signalling: an integrated mechanism regulating transitions between cell states. Bioessays. 2012; 34:110-8.

39. Shahi P, Seethammagari MR, Valdez JM, et al. Wnt and Notch pathways have interrelated opposing roles on prostate progenitor cell proliferation and differentiation. Stem Cells. 2011; 29:678-88.

40 Prieve MG, Guttridge KL, Munguia J, et al. Differential importin-a recognition and nuclear transport by nuclear localization signals within the high-mobility-group DNA binding domains of lymphoid enhancer factor 1 and T-cell factor 1. Mol Cell Biol. 1998; 18: 4819-32.

41. Yokoya F, Imamoto $\mathrm{N}$, Tachibana $\mathrm{T}$, et al. beta-catenin can be transported into the nucleus in a Ran-unassisted manner. Mol Biol Cell. 1999; 10:1119-31.

42. Asally M, Yoneda Y. Beta-catenin can act as a nuclear import receptor for its partner transcription factor, lymphocyte enhancer factor-1 (lef-1). Exp Cell Res. 2005; 308:357-63.

43. Ki H, Jung HC, Park JH, et al. Overexpressed LEF1 proteins display different nuclear localization patterns of beta-catenin in normal versus tumor cells. Cell Biol Int. 2006; 30: 253-61.

44. Xia J, Urabe K, Moroi $\mathrm{Y}$, et al. beta-Catenin mutation and its nuclear localization are confirmed to be frequent causes of Wnt signaling pathway activation in pilomatricomas. J Dermatol Sci. 2006; 41: 67-75. 